\title{
Acute kidney injury predicts poor left ventricular function for patients with peripartum cardiomyopathy
}

\author{
Jiajia Zhu and Wenxian Liu*
}

\begin{abstract}
Background: The aim of this study was to explore the risk factors associated with a poor left ventricular (LV) function among patients with peripartum cardiomyopathy (PPCM) and to determine the influence of acute kidney injury (AKI) on the LV function of the patients.

Methods: Sixty patients with PPCM were recruited between January 2007 and June 2018, among which 11 had AKI. The participants were divided into two groups, the recovery group (32 cases) and the nonrecovery group (28 cases), with their clinical features, echocardiography and electrocardiogram findings, laboratory results, and treatments compared between groups. We further determined the risk factors associated with nonrecovery and the influence posed by AKI on the LV function of the patients.
\end{abstract}

Results: Compared with the patients in the recovery group, those in the nonrecovery group had higher proportions of multiparity [78.6\% (22/28) vs. 43.8\% (14/32)], function class III- IV heart failure [92.9\% (26/28) vs. 71.9\% (23/32)], and a higher incidence of AKI [35.7\% (10/28) vs. 3.1\% (1/32)]. Logistic regression analysis showed that having AKI [odds ratio (OR): $10.556 ; 95 \%$ confidence interval (Cl) 1.177-94.654; $P=0.035$ ] and left ventricular ejection fraction (LVEF) < 40\% [OR: 4.533; 95\% Cl 1.118-18.382; $P=0.034$ ] were independently associated with nonrecovery of PPCM.

Conclusions: The prognosis of patients with PPCM and AKI during hospitalization was poor compared to those without AKI; therefore, clinicians should pay more attention to this phenomenon.

Keywords: Acute kidney injury, Left ventricular ejection fraction, Peripartum cardiomyopathy

\section{Background}

Peripartum cardiomyopathy (PPCM) is an idiopathic disease. Patients with PPCM present with clinical symptoms of heart failure secondary to left ventricular (LV) dysfunction towards the end of their pregnancy or during the postpartum period, mostly within months following delivery. PPCM is characterized echocardiographically by the presence of a LV ejection fraction (LVEF) $<45 \%$ and/or fractional shortening $<30 \%$ [1]. According to prior

\footnotetext{
*Correspondence: 18911662882@163.com
}

Department of Cardiology, Beijing Anzhen Hospital, Capital Medical

University, Beijing Institute of Heart, Lung and Blood Vessel Disease, No. 2

Anzhen Road, Beijing 100029, China studies, no other cause of heart failure has been found [1]. As an uncommon and potentially life-threatening illness, PPCM rarely has its cause(s) identified. Several risk factors for PPCM have been reported previously, including an advanced age, ethnicity (especially African), multiple gestations, hypertension during pregnancy, the LVEF and LV end diastolic diameter (LVEDD) at the diagnosis of PPCM, and the presence of LV thrombi [2-4]. However, it remains unclear whether more risk factors exist, and this is still the subject of ongoing debates.

As a complication of heart failure, acute kidney injury (AKI) has been shown to worsen the prognosis of patients with heart failure. Acute deterioration of renal function 
occurs in $20-40 \%$ of patients hospitalized for acute decompensated heart failure (ADHF) [5, 6]. Regardless of the timing of AKI onset, patients with ADHF and renal dysfunction have a significantly greater risk of rehospitalization and all-cause mortality compared to those without renal dysfunction [7]. In a retrospective study involving patients with heart failure, worsening renal function occurred in $11 \%$ of patients during hospitalization and in $16 \%$ of patients during the first 6 months after discharge [7]. Despite the established negative effects posed by AKI, the prognostic influence of AKI in patients with PPCM has not been described previously. Therefore, the aim of this study was to investigate the clinical features associated with PPCM and to determine the predictive value of in-hospital AKI for nonrecovery of PPCM among affected patients.

\section{Methods}

\section{Population and study design}

A total of 60 patients with PPCM admitted to Beijing Anzhen Hospital Affiliated to Capital Medical University were prospectively enrolled in this study from January 2007 to June 2018. The inclusion criteria were the presence of a PPCM diagnosis according to the PPCM Working Group of the European Society of Cardiology made by cardiologists and obstetricians; heart failure symptoms and a left ventricular ejection fraction (LVEF) below $45 \%$ at enrollment; an age of at least 18 years old; a heart failure severity of New York Heart Association (NYHA) functional class II-IV (for new patients only); and written informed consent. Patients were excluded from this study if they had a history of cardiomyopathy or severe organic valvular heart disease, had chronic hypertension, or failed to provide reliable contact phone numbers. The diagnosis of PPCM was made based on the diagnostic criteria endorsed by the European Society of Cardiology [1], including the following three criteria: first, the event occurred during the last month of pregnancy or within months following delivery; second, causes of heart failure other than PPCM could be excluded; finally, the echocardiographic findings of the index patients showed that LV systolic function was significantly impaired, manifesting as LVEF $<45 \%$ with or without LV enlargement. The demographic data, clinical features, laboratory and echocardiography parameters, and treatment received for the patients with PPCM were collected from their medical records. AKI was diagnosed according to the diagnostic criteria proposed by the Kidney Disease: Improving Global Outcomes (KDIGO) in 2012. Patients with a serum creatinine level $>1.5$ times their baseline value or an increase of $\geq 0.3 \mathrm{mg} / \mathrm{dL}(\geq 26.5 \mu \mathrm{mol} / \mathrm{L})$ within $48 \mathrm{~h}$ were diagnosed as having AKI [8].
All patients were followed up for at least 6 months after diagnosis. After follow-up, it was determined whether the participants' PPCM recovered, defined as LV systolic function improvement according to echocardiographic findings of LVEF $>50 \%$. On the contrary, nonrecovery of PPCM was identified if the patients had LVEF $\leq 50 \%$, received a heart transplantation, or died after 6 months of follow-up. The patients with PPCM were divided into the recovery group and the nonrecovery group, with the proportion of patients developing AKI documented. Finally, using logistic regression analysis, whether the presence of AKI was associated with nonrecovery among patients with PPCM was determined.

The Ethics Committee of Beijing Anzhen Hospital approved the current study (Approval No. 2019041X). Written informed consent was obtained from all individual participants included in this study.

\section{Statistical analysis}

All data were analyzed with SPSS 20.0 software (SPSS Inc., Chicago, IL, USA). Continuous variables were expressed as means \pm standard deviation, and categorical variables were expressed as percentages. Continuous variables were analyzed with the Student's t test or the Mann-Whitney U test. Categorical variables were compared using Pearson's chi-squared test. To assess the relationship between AKI and the prognosis of patients with PPCM, multivariate stepwise logistic regression analyses were conducted. A two-tailed $p$ value $<0.05$ was considered significant in all analyses.

\section{Results}

Sixty patients with PPCM were enrolled in this study, among whom $11(18.3 \%)$ had AKI. The mean age of these participants was $30 \pm 5$ years, and $36(60 \%)$ were multiparous. Three $(5 \%)$ of them had a history of PPCM. PPCM was diagnosed in 38 (63.3\%) participants before delivery and in 22 (36.7\%) participants during the postpartum period. With regard to their past medical history, $6(10.0 \%)$ and $4(6.7 \%)$ had hypertension and diabetes, respectively, while $12(20.0 \%)$ developed hypertension during their pregnancy, among whom $5(8.3 \%)$ had pregnancy-induced hypertension (PIH) and 7 (11.7\%) had preeclampsia. Their mean blood pressure (BP) during hospitalization was $116 \pm 18 / 77 \pm 14 \mathrm{mmHg}$, and their mean heart rate was $102 \pm 20$ beats per minute (bpm). Forty-four (73.3\%) had ST-T changes in their electrocardiograms. Their LVEDD and LVEF values were $59 \pm 8 \mathrm{~mm}$ and $37 \pm 13 \%$, respectively. Five (8.3\%) had intra-cardiac thrombosis. With regard to the treatments, all received diuretics, while 40 (66.7\%), 42 (70.0\%), 11 $(18.3 \%)$, and $3(5.0 \%)$ received angiotensin-converting enzyme inhibitors/angiotensin receptor blockers (ACEIs/ 
ARBs), $\beta$-blockers, anticoagulants, and vasoactive drugs including dopamine, epinephrine, and norepinephrine, respectively. Six $(10.0 \%)$ were treated with mechanical ventilation, among whom $4(6.7 \%)$ and $2(3.3 \%)$ received noninvasive and invasive ventilation, respectively. One patient was treated with intra-aortic balloon counter-pulsation (IABP) and extracorporeal membrane oxygenation (ECMO) (Table 1).

With regard to outcomes, 32 (53.3\%) participants recovered from PPCM, while 28 (46.7\%) did not. The mean age of the nonrecovered participants was older (nonrecovery vs. recovery, 32 vs. 29 years, $P=0.038$ ) than that of the recovered ones, and the proportion of multiparous participants was higher as well (nonrecovery vs. recovery, $78.6 \%$ vs. $43.8 \%, P=0.006$ ). There was no significant difference between groups regarding the past history of PPCM (nonrecovery vs. recovery, $7.1 \%$ vs. $3.1 \%, P=0.594)$, hypertension $(10.7 \%$ vs. 9.4\%, $P=1.000)$, diabetes $(10.7 \%$ vs. $3.1 \%, P=0.331)$, or PIH $(17.9 \%$ vs. $21.9 \%, P=0.698)$. The systolic BP was significantly lower in the nonrecovery group (nonrecovery vs. recovery, 111 vs. $122 \mathrm{mmHg}, P=0.02$ ), but the heart rates (105 vs. $99 \mathrm{bpm}, \mathrm{P}=0.270$ ) were comparable between groups. The nonrecovered participants had a significantly higher LVEDD (nonrecovery vs. recovery, 53 vs. $43 \mathrm{~mm}, P=0.003$ ) and a significantly lower LVEF ( $30 \%$ vs. $43 \%, P<0.001$ ) than the recovered ones, while the proportion of participants with intracardiac thrombosis $(14.3 \%$ vs. $3.1 \%, P=0.175)$ or electrocardiographic ST-T changes $(78.6 \%$ vs. $68.8 \%, P=0.391)$ did not differ. Regarding the laboratory tests, the nonrecovered participants had significantly higher serum creatinine (nonrecovery vs. recovery, 66.3 vs. $54.1 \mu \mathrm{mol} / \mathrm{L}, P<0.001)$ and brain natriuretic peptide (BNP) levels (1436 vs. $356 \mathrm{pg} /$ $\mathrm{mL}, P<0.001)$ on admission than the recovered ones. During hospitalization, all participants received diuretics. The proportion of digoxin users in the nonrecovery group was higher $(75.0 \%$ vs. $28.1 \%, P<0.001)$ than that in the recovery group, while there was no difference in the use of ACEIs/ARBs (nonrecovery vs. recovery, $75.0 \%$ vs. $59.4 \%, P=0.200)$, $\beta$-blockers $(75.0 \%$ vs. $65.6 \%, P=0.429)$, anticoagulants $(21.4 \%$ vs. $15.6 \%, P=0.562)$, vasoactive drugs $(7.1 \%$ vs. $3.1 \%, P=0.594)$, ventilation $(14.3 \%$ vs. $6.3 \%, P=0.404)$, IABP ( $3.6 \%$ vs. $0 \%, P=0.467)$, ECMO (3.6\% vs. $0 \%, P=0.467$ ), or continuous renal replacement therapy (CRRT) $(7.1 \%$ vs. $0 \%, P=0.214)$ between groups (Table 2).

All participants were divided into two groups: those with AKI (AKI group; 11 patients) and those without AKI (non-AKI group; 49 patients), according to the diagnostic criteria during hospitalization. There was no difference between those with and without AKI regarding age ( 32 vs. 30 years, $P=0.356)$, multiparity $(72.7 \%$
Table 1 Baseline characteristics of patients with PPCM $(n=60)$

\begin{tabular}{|c|c|}
\hline Characteristic & Value \\
\hline Age (years) & $30 \pm 5$ \\
\hline Primiparous (\%) & $24(40.0)$ \\
\hline Prenatal diagnosis (\%) & $38(63.3)$ \\
\hline Prenatal diagnosis (weeks) & $30 \pm 11$ \\
\hline Postnatal diagnosis (\%) & $22(36.7)$ \\
\hline Postnatal diagnosis (months) & $1(1-3)$ \\
\hline Cesarean section (\%) & $53(88.3)$ \\
\hline NYHA III-IV (\%) & $49(81.7)$ \\
\hline Hospitalization time (days) & $12(7-15)$ \\
\hline Hypertension (\%) & $6(10.0)$ \\
\hline Diabetes mellitus (\%) & $4(6.7)$ \\
\hline PPCM history (\%) & $3(5.0)$ \\
\hline Pregnancy-induced hypertension syndrome (\%) & $5(8.3)$ \\
\hline Preeclampsia (\%) & $7(11.7)$ \\
\hline $\mathrm{SBP}(\mathrm{mmHg})$ & $116 \pm 18$ \\
\hline $\mathrm{DBP}(\mathrm{mmHg})$ & $77 \pm 14$ \\
\hline $\mathrm{HR}(\mathrm{bpm})$ & $102 \pm 20$ \\
\hline \multicolumn{2}{|l|}{ Echocardiographic findings } \\
\hline $\operatorname{LVEF}(\%)$ & $37 \pm 13$ \\
\hline Left ventricular thrombosis (\%) & $5(8.3)$ \\
\hline $\operatorname{LVEDD}(\mathrm{mm})$ & $59 \pm 8$ \\
\hline \multicolumn{2}{|l|}{ Electrocardiographic findings } \\
\hline ST-T changes (\%) & $44(73.3)$ \\
\hline QRS time (ms) & $94.2 \pm 21.5$ \\
\hline QTc time (ms) & $475 \pm 29$ \\
\hline Hemoglobin (g/L) & $123 \pm 16$ \\
\hline Platelet (G/L) & $262 \pm 84$ \\
\hline $\operatorname{ALT}(U / L)$ & $22(12-46)$ \\
\hline AST (U/L) & $24(19-34)$ \\
\hline Serum creatinine $(\mu \mathrm{mol} / \mathrm{L})$ & $64.2(54.1-69.3)$ \\
\hline $\mathrm{BNP}(\mathrm{pg} / \mathrm{mL})$ & $819(378-2143)$ \\
\hline AKI (\%) & $11(18.3)$ \\
\hline Ventilator (\%) & $6(10.0)$ \\
\hline Noninvasive ventilator (\%) & $4(6.7)$ \\
\hline Invasive mechanical ventilator (\%) & $2(3.3)$ \\
\hline IABP (\%) & $1(1.7)$ \\
\hline ECMO (\%) & $1(1.7)$ \\
\hline CRRT (\%) & $2(3.3)$ \\
\hline Diuretic (\%) & $60(100)$ \\
\hline ACEI/ARB (\%) & $40(66.7)$ \\
\hline$\beta$-Blockers (\%) & $42(70.0)$ \\
\hline Ivabradine (\%) & $3(5.0)$ \\
\hline Anticoagulation (\%) & $11(18.3)$ \\
\hline Digoxin (\%) & $30(50.0)$ \\
\hline Vasoactive agents (\%) & $3(5.0)$ \\
\hline
\end{tabular}

NYHA New York Heart Association, PPCM peripartum cardiomyopathy, SBP systolic blood pressure, DBP diastolic blood pressure, $H R$ heart rate, $L V E F$ left ventricular ejection fraction, $L V E D D$ left ventricular end-diastolic diameter, $A L T$ alanine transaminase, AST aspartate transaminase, AKI acute kidney injury, $I A B P$ intra-aortic balloon pump, $E C M O$ extracorporeal membrane oxygenation, CRRT continuous renal replacement therapy, ACEI/ARB angiotensin-converting enzyme inhibitor/angiotensin receptor blocker 
Table 2 Comparison of the clinical and laboratory characteristics between the recovery and the nonrecovery groups $(n=60)$

\begin{tabular}{|c|c|c|c|}
\hline Characteristic & Recovery Group ( $n=32$ ) & Nonrecovery Group $(n=28)$ & $P$ value \\
\hline AKI (\%) & $1(3.1)$ & $10(35.7)$ & 0.002 \\
\hline Age (years) & $29 \pm 4$ & $32 \pm 6$ & 0.038 \\
\hline Primiparous (\%) & $14(43.8)$ & $22(78.6)$ & 0.006 \\
\hline Cesarean section (\%) & $28(87.5)$ & $28(100)$ & 0.116 \\
\hline NYHA III-IV (\%) & $23(71.9)$ & $26(92.9)$ & 0.048 \\
\hline Hospitalization time (days) & $12(8-15)$ & $11(6-15)$ & 0.542 \\
\hline Hypertension (\%) & $3(9.4)$ & $3(10.7)$ & 1.000 \\
\hline Diabetes mellitus (\%) & $1(3.1)$ & $3(10.7)$ & 0.331 \\
\hline PPCM history (\%) & $1(3.1)$ & $2(7.1)$ & 0.594 \\
\hline Pregnancy-induced hypertension syndrome (\%) & $2(6.3)$ & $3(10.7)$ & 0.657 \\
\hline Preeclampsia (\%) & $5(15.6)$ & $2(7.1)$ & 0.432 \\
\hline $\mathrm{SBP}(\mathrm{mmHg})$ & $122 \pm 16$ & $111 \pm 17$ & 0.02 \\
\hline $\mathrm{DBP}(\mathrm{mmHg})$ & $79 \pm 11$ & $74 \pm 15$ & 0.1 \\
\hline $\mathrm{HR}(\mathrm{bpm})$ & $99 \pm 19$ & $105 \pm 20$ & 0.27 \\
\hline \multicolumn{4}{|l|}{ Echocardiographic findings } \\
\hline LVEF (\%) & $43 \pm 12$ & $30 \pm 10$ & $<0.001$ \\
\hline Left ventricular thrombosis (\%) & $1(3.1)$ & $4(14.3)$ & 0.175 \\
\hline $\operatorname{LVEDD~(mm)~}$ & $43 \pm 8$ & $53 \pm 8$ & 0.003 \\
\hline \multicolumn{4}{|l|}{ Electrocardiographic findings } \\
\hline ST-T changes (\%) & $22(68.8)$ & $22(78.6)$ & 0.391 \\
\hline QRS time (ms) & $94 \pm 26$ & $95 \pm 14$ & 0.808 \\
\hline QTc time (ms) & $463 \pm 42$ & $457 \pm 39$ & 0.64 \\
\hline Hemoglobin (g/L) & $120 \pm 13$ & $126 \pm 19$ & 0.136 \\
\hline Platelet (G/L) & $250 \pm 80$ & $276 \pm 87$ & 0.25 \\
\hline $\operatorname{ALT}(\mathrm{U} / \mathrm{L})$ & $20(10-35)$ & $23(16-63)$ & 0.11 \\
\hline AST (U/L) & $22(16-30)$ & $26(21-45)$ & 0.069 \\
\hline Serum creatinine $(\mu \mathrm{mol} / \mathrm{L})$ & $54.1(44-65.42)$ & $66.3(56.87-83.25)$ & $<0.001$ \\
\hline $\mathrm{BNP}(\mathrm{pg} / \mathrm{mL})$ & $356(67-819)$ & $1436(696-2353)$ & $<0.001$ \\
\hline Ventilator (\%) & $2(6.3)$ & $4(14.3)$ & 0.404 \\
\hline IABP (\%) & 0 & $1(3.6)$ & 0.467 \\
\hline ECMO (\%) & 0 & $1(3.6)$ & 0.467 \\
\hline CRRT (\%) & 0 & $2(7.1)$ & 0.214 \\
\hline Diuretic (\%) & $32(100)$ & $28(100)$ & 1.000 \\
\hline ACEI/ARB (\%) & 19 (59.4) & $21(75.0)$ & 0.200 \\
\hline$\beta$-blockers (\%) & $21(65.6)$ & $21(75.0)$ & 0.429 \\
\hline Ivabradine (\%) & $2(6.3)$ & $1(3.6)$ & 1.000 \\
\hline Anticoagulation (\%) & $5(15.6)$ & $6(21.4)$ & 0.562 \\
\hline Digoxin (\%) & $9(28.1)$ & $21(75.0)$ & $<0.001$ \\
\hline Vasoactive agents (\%) & $1(3.1)$ & $2(7.1)$ & 0.594 \\
\hline
\end{tabular}

NYHA New York Heart Association, PPCM peripartum cardiomyopathy, SBP systolic blood pressure, DBP diastolic blood pressure, HR heart rate, $L V E F$ left ventricular ejection fraction, $L V E D D$ left ventricular end-diastolic diameter, $A L T$ alanine transaminase, $A S T$ aspartate transaminase, $A K I$ acute kidney injury, IABP intra-aortic balloon pump, ECMO extracorporeal membrane oxygenation, CRRT continuous renal replacement therapy, $A C E I / A R B$ angiotensin-converting enzyme inhibitor/angiotensin receptor blocker

vs. $57.1 \%, P=0.34)$, a past history of PPCM ( $0 \%$ vs. $6.1 \%, P=1.000)$, hypertension ( $0 \%$ vs. $12.2 \%, P=0.221)$, diabetes ( $0 \%$ vs. $8.2 \%, P=1.000)$, PIH (27.3\% vs. $18.4 \%$, $P=0.677)$, systolic BP $(112 \pm 20$ vs. $118 \pm 17 \mathrm{mmHg}$, $P=0.334)$, diastolic BP $(79 \pm 16$ vs. $76 \pm 13 \mathrm{mmHg}$, $P=0.52)$, mean heart rate (106 vs. $101 \mathrm{bpm}, P=0.408)$, or ST-T changes (90.9\% vs. $69.4 \%, P=0.259$ ). However, the participants with AKI had a significantly lower LVEF (AKI vs. non-AKI, $29 \%$ vs. 38\%, $P=0.026$ ) and a higher proportion of intra-cardiac thrombosis (27.3\% vs. $4.1 \%, P=0.039)$ than those without. With regard to the laboratory results, the levels of serum 
creatinine (79.5 vs. $55.5 \mu \mathrm{mol} / \mathrm{L}, P<0.05)$ and BNP (2243 vs. $546 \mathrm{pg} / \mathrm{mL}, P<0.05)$ in the AKI group were significantly greater than those in the non-AKI group on admission. During hospitalization, the proportions of digoxin ( $90.9 \%$ vs. $40.8 \%, P=0.006)$ and anticoagulant users $(54.5 \%$ vs. $10.2 \%, P=0.001)$ were greater in the AKI group than in the non-AKI group. Meanwhile, there was no difference between groups regarding the proportion of those requiring ACEI/ARB (63.6\% vs. $67.3 \%, P=1.000), \beta$-blockers $(54.5 \%$ vs. $73.5 \%$, $P=0.216)$, vasoactive drugs $(18.2 \%$ vs. $2.0 \%, P=0.084)$, ventilation $(27.3 \%$ vs. $6.1 \%, P=0.069)$, IABP $(9.1 \%$ vs. $0 \%, P=0.183)$, or ECMO ( $9.1 \%$ vs. $0 \%, P=0.183)$. Two patients with AKI received CRRT (Table 3).

Table 3 Comparison of the clinical characteristics between patients with and without AKI $(n=60)$

\begin{tabular}{|c|c|c|c|}
\hline Characteristic & $A K I(n=11)$ & Non-AKI $(n=49)$ & $P$ value \\
\hline Age (years) & $32 \pm 6$ & $30 \pm 5$ & 0.356 \\
\hline Primiparous (\%) & $8(72.7)$ & $28(57.1)$ & 0.34 \\
\hline Cesarean section (\%) & $11(100.0)$ & $43(91.8)$ & 1.000 \\
\hline NYHA III-IV (\%) & $11(100)$ & $38(77.6)$ & 0.189 \\
\hline Hospitalization time (days) & $14(8-23)$ & $11(7-15)$ & 0.105 \\
\hline Hypertension (\%) & 0 & $6(12.2)$ & 0.221 \\
\hline Diabetes mellitus (\%) & 0 & $4(8.2)$ & 1.000 \\
\hline PPCM history (\%) & 0 & $3(6.1)$ & 1.000 \\
\hline Pregnancy-induced hypertension syndrome (\%) & 0 & $5(10.2)$ & 0.573 \\
\hline Preeclampsia (\%) & $3(27.3)$ & $4(8.2)$ & 0.108 \\
\hline $\mathrm{SBP}(\mathrm{mmHg})$ & $112 \pm 20$ & $118 \pm 17$ & 0.334 \\
\hline $\mathrm{DBP}(\mathrm{mmHg})$ & $79 \pm 16$ & $76 \pm 13$ & 0.52 \\
\hline $\mathrm{HR}(\mathrm{bpm})$ & $106 \pm 19$ & $101 \pm 20$ & 0.408 \\
\hline \multicolumn{4}{|l|}{ Echocardiographic findings } \\
\hline LVEF (\%) & $29 \pm 11$ & $38 \pm 13$ & 0.026 \\
\hline Left ventricular thrombosis (\%) & $3(27.3)$ & $2(4.1)$ & 0.039 \\
\hline LVEDD (mm) & $52 \pm 8$ & $47 \pm 9$ & 0.386 \\
\hline \multicolumn{4}{|l|}{ Electrocardiographic findings } \\
\hline ST-T changes (\%) & $10(90.9)$ & $34(69.4)$ & 0.259 \\
\hline QRS time (ms) & $88 \pm 10$ & $96 \pm 23$ & 0.397 \\
\hline QTc time (ms) & $457 \pm 54$ & $460 \pm 37$ & 0.803 \\
\hline Hemoglobin (g/L) & $126 \pm 17$ & $122 \pm 16$ & 0.485 \\
\hline Platelet (G/L) & $238 \pm 82$ & $267 \pm 84$ & 0.3 \\
\hline $\operatorname{ALT}(U / L)$ & $26(13-83)$ & $21(12-43)$ & 0.233 \\
\hline $\mathrm{AST}(\mathrm{U} / \mathrm{L})$ & $27(25.3-29.4)$ & $22(16-31)$ & 0.097 \\
\hline Serum creatinine $(\mu \mathrm{mol} / \mathrm{L})$ & $79.5(63.4-98.3)$ & $55.5(52.95-65.75)$ & 0.016 \\
\hline $\mathrm{BNP}(\mathrm{pg} / \mathrm{mL})$ & $2243(1272-5000)$ & $546.55(104.63-1403.75)$ & 0.001 \\
\hline Ventilator (\%) & $3(27.3)$ & $3(6.1)$ & 0.069 \\
\hline IABP (\%) & $1(9.1)$ & 0 & 0.183 \\
\hline ECMO (\%) & $1(9.1)$ & 0 & 0.183 \\
\hline CRRT (\%) & $2(18.2)$ & 0 & 0.031 \\
\hline Diuretic (\%) & $11(100)$ & $49(100)$ & 1.000 \\
\hline ACEI/ARB (\%) & $7(63.6)$ & $33(67.3)$ & 1.000 \\
\hline$\beta$-blockers (\%) & $6(54.5)$ & $36(73.5)$ & 0.216 \\
\hline Ivabradine (\%) & 0 & $3(6.1)$ & 1.000 \\
\hline Anticoagulation (\%) & $6(54.5)$ & $5(10.2)$ & 0.001 \\
\hline Digoxin (\%) & $10(90.9)$ & $20(40.8)$ & 0.006 \\
\hline Vasoactive agents (\%) & $2(18.2)$ & $1(2.0)$ & 0.084 \\
\hline
\end{tabular}

NYHA New York Heart Association, PPCM peripartum cardiomyopathy, SBP systolic blood pressure, DBP diastolic blood pressure, $H R$ heart rate, $L V E F$ left ventricular ejection fraction, $L V E D D$ left ventricular end-diastolic diameter, $A L T$ alanine transaminase, $A S T$ aspartate transaminase, $A K I$ acute kidney injury, IABP intra-aortic balloon pump, ECMO extracorporeal membrane oxygenation, CRRT continuous renal replacement therapy, $A C E I / A R B$ angiotensin-converting enzyme inhibitor/angiotensin receptor blocker 
Finally, logistic regression analysis was used to determine the risk factors associated with nonrecovery among all participants with PPCM. Having AKI [odds ratio (OR): 10.556; 95\% confidence interval (CI): 1.177-94.654; $P=0.035$ ] or an EF $<40 \%$ (OR: 4.533 ; 95\% CI 1.11818.382; $P=0.034$ ) was found to be independently associated with nonrecovery of PPCM (Table 4).

\section{Discussion}

In this study, the incidence of AKI among patients with PPCM was found to be $18.3 \%$. In addition, AKI was determined to be an independent risk factor associated with nonrecovery in these patients.

PPCM is a type of cardiomyopathy that mainly occurs late during pregnancy or during the first postpartum month. The clinical manifestations and the principles of management of PPCM are similar to those of dilated cardiomyopathy. However, PPCM is unique because it only involves women during their pregnancy or following delivery. The incidence of PPCM differs between countries. In Haiti, the incidence of PPCM is about 1:300 [2]; while in South Africa and United States, it is approximately 1:1000 [3, 9]. In Japan, the incidence of PPCM is only 1:20,000 [10]. With evolution of the definition of PPCM and the increased awareness of this disease, it is now understood that the complications associated with PPCM are devastating. To the best of our knowledge, there is currently a lack of large cohort studies of PPCM in China. A study involving pregnant women treated at Peking Union Medical College Hospital between January 1995 and December 2014 showed that only one maternal morbidity event was noted per 1067 deliveries [11]. Although PPCM is a rare disease, it poses a serious threat to the health of pregnant women and their newborns. One report has suggested that once pregnant women are diagnosed with PPCM, their mortality rate could be as high as $16 \%$ [12-14]. In addition, Karaye et al. found that the mortality rate was $18.7 \%$ in Nigeria in the largest PPCM cohort studied to date $[15,16]$. Therefore, it is necessary to gain more understanding regarding the etiologies and risk factors of mortality for patients with PPCM.
The exact etiology of PPCM is still unclear, but the onset of PPCM may be associated with changes in hormone levels, the hemodynamic status, and metabolic states during the perinatal period [17]. Prior studies have further demonstrated that an older age, multiple pregnancies, multiparity, PIH, preeclampsia, and a low socioeconomic status are all risk factors for developing PPCM $[17,18]$. In the current study of 60 patients with PPCM, the mean age at delivery of these women was 30 years old. About $60 \%$ of the participants had delivered more than once, and nearly $20 \%$ had PIH during pregnancy. Therefore, the clinical features of the patients enrolled in this study are consistent with the findings described in the existing literature.

The prognosis of PPCM varies substantially among affected women. It is generally believed that the LVEF of patients with PPCM will return to normal within 6 months after onset. If their LVEF does not recover 6 months later, it is likely that the cardiac damage has become irreversible in these patients and can culminate in mortality [19]. After follow-up, 46.7\% of the patients with PPCM did not recover. The patients with nonrecovered PPCM were older than those who had recovered, consistent with the findings of patients who are susceptible to PPCM development. In addition, the patients with nonrecovered PPCM had a lower systolic BP and LVEF at admission but a higher LVEDD, serum creatinine level, and BNP level than those with recovered PPCM. Moreover, the incidence of AKI was higher in those with nonrecovered PPCM, suggesting that worse outcomes occur more frequently in participants with severe hemodynamic disturbances at admission.

AKI has already been established as a risk factor for a poor prognosis in patients with heart failure. The incidence rate of AKI in patients with ADHF is 20-45\%, according to different AKI definitions [8, 20, 21], and the presence of AKI results in a $22 \%$ increase of mortality. However, no previous studies have addressed AKI and its influence on patients with PPCM. In this study, the incidence of AKI in patients with PPCM was $18.3 \%$ during hospitalization. Furthermore, the clinical features between the patients with and without AKI were similar except for a lower LVEF in those with AKI, indicating

Table 4 Univariate and multivariate logistic regression analyses of factors associated with nonrecovered peripartum cardiomyopathy

\begin{tabular}{|c|c|c|c|c|c|c|}
\hline Parameter & OR & $95 \% \mathrm{Cl}$ & $P$ value & OR & $95 \% \mathrm{Cl}$ & $P$ value \\
\hline AKI & 17.222 & $2.034-145.810$ & 0.009 & 10.556 & $1.177-94.654$ & 0.035 \\
\hline NYHA III-IV & 5.087 & $0.995-26.006$ & 0.051 & & & \\
\hline LVEF $<40 \%$ & 7.714 & $2.170-27.419$ & 0.002 & 4.533 & $1.118-18.382$ & 0.034 \\
\hline LVEDD > $55 \mathrm{~mm}$ & 4.714 & $1.506-14.760$ & 0.008 & 2.758 & $0.733-10.377$ & 0.134 \\
\hline
\end{tabular}

$A K I$ acute kidney injury, $C l$ confidence interval, $L V E F$ left ventricular ejection fraction, $O R$ odds ratio 
that $\mathrm{AKI}$ is not related to drug use but to the disease per se. In the existing literature, AKI is an independent risk factor for a poor prognosis of patients with ADHF that is independent of a reduced ejection fraction in patients with HFrEF or those with a mid-range ventricular ejection fraction (HFmrEF) [22]. On the other hand, PPCM is a special disease entity distinct from heart failure, and it deserves separate attention. We found that AKI was an independent risk factor for a poor prognosis, regardless of EF, LVEDD, and BNP based on the logistic regression results. Regarding the relationships between AKI, EF, LVEDD, and BNP, those in the AKI group had a lower EF, a larger LVEDD, and a higher BNP than those without. These findings suggest that those with AKI had deteriorations in cardiac function [23, 24]. We believe that the patients in the AKI group had a lower EF, a larger LVEDD, and a higher BNP level, indicating that the condition of PPCM patients is critical. Therefore, we should pay more attention to the influence of AKI in patients with PPCM.

In addition, we found that the serum creatinine level was $79.5 \mu \mathrm{mol} / \mathrm{L}$ in patients with AKI and $55.5 \mu \mathrm{mol} / \mathrm{L}$ in those without, and the hemoglobin level was $126 \pm 17 \mathrm{~g} / \mathrm{L}$ in those with AKI and $122 \pm 16 \mathrm{~g} / \mathrm{L}$ in those without. Patients diagnosed as having chronic renal insufficiency are more likely to have anemia due to reduced EPO levels. However, this change in hemoglobin levels does not necessarily exist in patients with AKI because PPCM frequently presents as acute heart failure. Meanwhile, patients in the AKI group had a critical clinical condition because of their low EF value, larger LVEDD, and high BNP levels. It is likely that the patients received an increased dose of multiple medications to alleviate symptoms, leading to hemoconcentration and higher hemoglobin levels. In our study, although the patients in the AKI group had increased creatinine and hemoglobin levels, there was no statistical difference in the hemoglobin levels between the two groups. A larger sample size is needed to draw a definite conclusion.

In this study, LVEF $<40 \%$ and AKI were both determined to be independent risk factors for nonrecovered PPCM. Similarly, Chapa et al. identified that LVEDD $>60 \mathrm{~mm}$ and $\mathrm{LVEF}<20 \%$ were independent risk factors for nonrecovery of cardiac function in 32 patients with PPCM [25]. Goland et al. also found that LVEF $<25 \%$ was predictive of severe cardiovascular events in patients with PPCM [5]. In the PEACE register study [15], the researchers consecutively recruited 244 PPCM patients from 14 sites in Nigeria and applied a structured follow-up for a median of 17 months. They found that a maternal age $<20$ years [hazard ratio (HR): 2.40, 95\% CI 1.27-4.54], hypotension (HR: 1.87, 95\% CI 1.02-3.43), tachycardia (HR: $2.38,95 \%$ CI 1.05-5.43), and LVEF $<25 \%$ at baseline (HR: 2.11, 95\% CI 1.12-3.95) could predict mortality. These results are consistent with ours. However, their finding that the regular use of betablockers at the 6-month follow-up (HR: 0.20, 95\% CI 0.09-0.41) was independently associated with a lower mortality risk was not found in our study. This may be related to the fact that beta-blockers were used according to the local guidelines for heart failure and PPCM only when needed. AKI at admission was also determined to be an independent risk factor for a poor prognosis. This is due to the fact that the kidney is susceptible to alterations in the perfusion pressure. For example, when hemodynamic disturbance occurs, the renal blood flow is compromised, followed by a decline in the glomerular filtration rate, renal perfusion, and the creatinine clearance rate. Therefore, more attention should be paid to patients with PPCM when AKI occurs during their hospitalization.

\section{Limitation}

This investigation was a single-center study with a small sample size. The data were retrospectively collected from medical reports, and the echocardiographic findings were provided by different examiners. In addition, some of the participants had incomplete data because of the prolonged follow-up duration. However, with improvements in the diagnostic criteria of PPCM, the incidence of PPCM is expected to increase. In the future, a multicenter study will be needed to analyze the etiology and the risk factors for nonrecovery in these patients.

\section{Conclusion}

The findings of this study suggested that AKI at admission was significantly associated with nonrecovered PPCM. If AKI occurs in patients with PPCM during their hospitalization, they tend to have a poor recovery of cardiac function, leading to long-term adverse outcomes. These changes in renal function were not caused by drug use. Therefore, clinicians should pay more attention to AKI in patients with PPCM.

\section{Abbreviations}

PPCM: Peripartum cardiomyopathy; AKI: Acute kidney injury; NYHA: New York Heart Association; BNP: B-type natriuretic peptide; LVEDD: End-diastolic diameter of the left ventricle; LVEF: Left ventricular ejection fraction; OR: Odds ratio; Cl: Confidence interval; LV: Left ventricular; ADHF: Acute decompensated heart failure; $\mathrm{PIH}$ : Pregnancy-induced hypertension; BP: Blood pressure; bpm: Beats per minute; ACEls/ARBs: Angiotensin-converting enzyme inhibitors/ angiotensin receptor blockers; IABP: Intra-aortic balloon counter-pulsation; ECMO: Extracorporeal membrane oxygenation; SBP: Systolic blood pressure; DBP: Diastolic blood pressure; HR: Heart rate; ALT: Alanine transaminase; AST: Aspartate transaminase; CRRT: Continuous renal replacement therapy.

Acknowledgements

None. 


\section{Authors' contributions}

JZ participated in the trial design, collected and analyzed the data, and wrote the article. WL is the corresponding author who participated in the trial design and reviewed the article. All authors read and approved the final manuscript.

\section{Funding}

This work was supported by the Beijing Health and Technology Achievements for Technology Promotion Project (TG-2017-34). The authors declare that the funding body was not involved in study design, data collection, analysis, interpretation, or writing.

\section{Availability of data and materials}

The datasets generated and analyzed during the present study are available from the corresponding author on reasonable request.

\section{Declarations}

Ethics approval and consent to participate

This study was approved by the Ethics Committee of Beijing Anzhen Hospital (Approval No. 2019041X). All procedures performed in studies involving human participants were in accordance with the ethics standards of the institutional and national research committee and with the 1964 Helsinki Declaration and its later amendments or comparable ethics standards. Written informed consent was obtained from all individual participants included in this study.

\section{Consent for publication}

Not applicable.

\section{Competing interests}

The authors declare that they have no competing interests.

Received: 11 January 2021 Accepted: 14 April 2021

Published online: 22 April 2021

\section{References}

1. Sliwa K, Hilfiker-Kleiner D, Petrie MC, Mebazaa A, Pieske B, Buchmann $E$, et al. Current state of knowledge on aetiology, diagnosis, management, and therapy of peripartum cardiomyopathy: a position statement from the Heart Failure Association of the European Society of Cardiology Working Group on peripartum cardiomyopathy. Eur J Heart Fail. 2010;12:767-78.

2. Isogai T, Kamiya CA. Worldwide incidence of peripartum cardiomyopathy and overall maternal mortality. Int Heart J. 2019:60:503-11.

3. Honigberg MC, Givertz MM. Peripartum cardiomyopathy. BMJ. 2019;364:k5287.

4. Kolte D, Khera S, Aronow WS, Palaniswamy C, Mujib M, Ahn C, et al. Temporal trends in incidence and outcomes of peripartum cardiomyopathy in the United States: a nationwide population-based study. J Am Heart Assoc. 2014;3:e001056.

5. Forman DE, Butler J, Wang Y, Abraham WT, O'Connor CM, Gottlieb SS, et al. Incidence, predictors at admission, and impact of worsening renal function among patients hospitalized with heart failure. J Am Coll Cardiol. 2004;43:61-7.

6. Rastogi A, Fonarow GC. The cardiorenal connection in heart failure. Curr Cardiol Rep. 2008;10:190-7.

7. Damman K, Jaarsma T, Voors AA, Navis G, Hillege HL, van Veldhuisen DJ. Both in- and out-hospital worsening of renal function predict outcome in patients with heart failure: results from the Coordinating Study Evaluating Outcome of Advising and Counseling in Heart Failure (COACH). Eur J Heart Fail. 2009;11:847-54.
8. Acute Kidney Injury Work Group. Kidney Disease: Improving Global Outcomes (KDIGO) — clinical practice guideline for acute kidney injury. Kidney Int. 2012;2:1-138.

9. McNamara DM, Elkayam U, Alharethi R, Damp J, Hsich E, Ewald G, et al. Clinical outcomes for peripartum cardiomyopathy in North America: results of the IPAC study (investigations of pregnancy-associated cardiomyopathy). J Am Coll Cardiol. 2015:66:905-14.

10. Guo X, Liu Y, Fang L. Fang Q [The clinical characteristics and prognosis of patients with peripartum cardiomyopathy]. Zhonghua Nei Ke Za Zhi. 2016;55:127-30.

11. Asad ZUA, Maiwand M, Farah F, Dasari TW. Peripartum cardiomyopathy: a systematic review of the literature. Clin Cardiol. 2018;41:693-7.

12. Azibani F, Sliwa K. Peripartum cardiomyopathy: an update. Curr Heart Fail Rep. 2018;15:297-306.

13. Yaméogo NV, Samadoulougou AK, Kagambèga LJ, Kologo KJ, Millogo GRC, Thiam A, et al. Maternal and fetal prognosis of subsequent pregnancy in black African women with peripartum cardiomyopathy. BMC Cardiovasc Disord. 2018;18:119.

14. Moulig V, Pfeffer TJ, Ricke-Hoch M, Schlothauer S, Koenig T, Schwab J, et al. Long-term follow-up in peripartum cardiomyopathy patients with contemporary treatment: low mortality, high cardiac recovery, but significant cardiovascular co-morbidities. Eur J Heart Fail. 2019;21:1534-42.

15. Karaye KM, Sa'idu H, Balarabe SA, Ishaq NA, Adamu UG, Mohammed IY, et al. Clinical features and outcomes of peripartum cardiomyopathy in Nigeria. J Am Coll Cardiol. 2020;76:2352-64.

16. Karaye KM, Lindmark K, Henein M. Right ventricular systolic dysfunction and remodelling in Nigerians with peripartum cardiomyopathy: a longitudinal study. BMC Cardiovasc Disord. 2016;16:27.

17. Ersbøll AS, Damm P, Gustafsson F, Vejlstrup NG, Johansen M. Peripartum cardiomyopathy: a systematic literature review. Acta Obstet Gynecol Scand. 2016:95:1205-19.

18. Blauwet LA, Libhaber E, Forster O, Tibazarwa K, Mebazaa A, Hilfiker-Kleiner $D$, et al. Predictors of outcome in 176 South African patients with peripartum cardiomyopathy. Heart. 2013;99:308-13.

19. Mehta RL, Kellum JA, Shah SV, Molitoris BA, Ronco C, Warnock DG, et al. Acute Kidney Injury Network: report of an initiative to improve outcomes in acute kidney injury. Crit Care. 2007;11:R31.

20. Ekizler FA, Cay S, Kafes H, Ozeke O, Ozcan F, Topaloglu S, et al. The prognostic value of positive T wave in lead aVR: A novel marker of adverse cardiac outcomes in peripartum cardiomyopathy. Ann Noninvasive Electrocardiol. 2019;24:e12631.

21. Biteker M, Özlek B, Özlek E, Çil C, Çelik O, Doğan V, et al. Predictors of early and delayed recovery in peripartum cardiomyopathy: a prospective study of 52 Patients. J Matern Fetal Neonatal Med. 2020;33:390-7.

22. Lala RI, Lungeanu D, Puschita M, Pop-Moldovan A, Darabantiu D. Acute kidney injury: a clinical issue in hospitalized patients with heart failure with mid-range ejection fraction. Pol Arch Intern Med. 2018;128:746-54

23. Shirakabe A, Hata N, Kobayashi N, Shinada T, Tomita K, Tsurumi M, et al. Prognostic impact of acute kidney injury in patients with acute decompensated heart failure. Circ J. 2013;77:687-96.

24. Shirakabe A, Hata N, Kobayashi N, Okazaki H, Matsushita M, Shibata Y, et al. Worsening renal failure in patients with acute heart failure: the importance of cardiac biomarkers. ESC Heart Fail. 2019;6:416-27.

25. Stevens PE, Levin A. Evaluation and management of chronic kidney disease: synopsis of the kidney disease: improving global outcomes 2012 clinical practice guideline. Ann Intern Med. 2013;158:825-30.

\section{Publisher's Note}

Springer Nature remains neutral with regard to jurisdictional claims in published maps and institutional affiliations. 